\title{
The Alzheimer's Prevention Registry: A Large Internet-Based Participant Recruitment Registry to Accelerate Referrals to Alzheimer's- Focused Studies
}

\author{
J.B. Langbaum ${ }^{1,5,8}$, N. High ${ }^{1}$, J. Nichols ${ }^{1,2}$, C. Kettenhoven ${ }^{1}$, E.M. Reiman ${ }^{* 1,1,4,6,6,7,8}$, P.N. Tariot ${ }^{*}, 1,4,8$
}

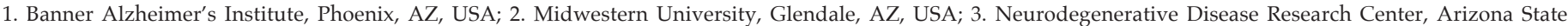

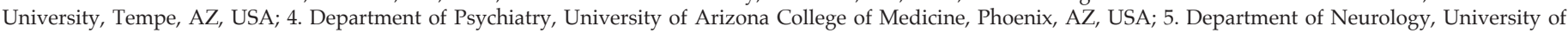

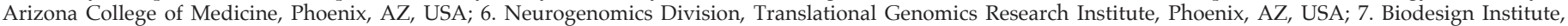
Arizona State University, Tempe, AZ, USA; 8. Arizona Alzheimer's Consortium, Phoenix, AZ, USA; * co-senior authors.

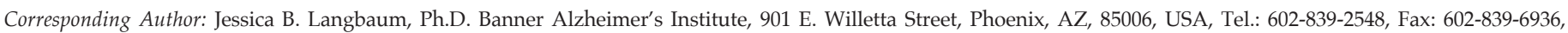
Email: Jessica.Langbaum@bannerhealth.com

J Prev Alz Dis 2020;4(7):242-250

Published online May 25, 2020, http:/ / dx.doi.org/10.14283/jpad.2020.31

\begin{abstract}
BACKGROUND: Recruitment for Alzheimer's disease (AD)focused studies, particularly prevention studies, is challenging due to the public's lack of awareness about study opportunities coupled with studies' inclusion and exclusion criteria, resulting in a high screen fail rate.

OBJECTIVES: To develop an internet-based participant recruitment registry for efficiently and effectively raising awareness about AD-focused study opportunities and connecting potentially eligible volunteers to studies in their communities.

METHODS: Individuals age 18 and older are eligible to join the Alzheimer's Prevention Registry (APR). Individuals provide first and last name, year of birth, country, and zip/postal code to join the APR; for questions regarding race, ethnicity, sex, family history of AD or other dementia, and diagnosis of cognitive impairment, individuals have the option to select "prefer not to answer." The APR website maintains a list of recruiting studies and contacts members who have opted in by email when new studies are available for enrollment.

RESULTS: As of December 1, 2019, 346,661 individuals had joined the APR. Members had a mean age of 63.3 (SD 11.7) years and were predominately women (75\%). 94\% were cognitively unimpaired, $50 \%$ reported a family history of AD or other dementia, and of those who provided race, $76 \%$ were white. $39 \%$ joined the APR as a result of a paid social media advertisement. To date, the APR helped recruit for 82 studies. CONCLUSIONS: The APR is a large, internet-based participant recruitment registry designed to raise awareness about $A D$ prevention research and connect members with enrolling studies in their communities. It has demonstrated the ability to recruit and engage a large number of highly motivated members and assist researchers in meeting their recruitment goals. Future publications will report on the effectiveness of APR for accelerating recruitment and enrollment into AD-focused studies.
\end{abstract}

Key words: Registry, recruitment, Alzheimer's disease.

\section{Introduction}

A lzheimer's disease (AD) remains one of the greatest medical, economic, and societal burdens in the United States (US) and globally (1). In the US, an estimated 5.7 million people are living with dementia due to $\mathrm{AD}$, a number projected to reach 13.8 million by 2050 barring a medical breakthrough (2). Interventions that delay the symptomatic onset of $\mathrm{AD}$ by even by 1 or 2 years would have a major public health impact (3). With a heightened sense of urgency, numerous AD prevention studies are underway, with many more planned.

The sharp growth in AD prevention trials requires an unprecedented screening and enrollment funnel. Specifically, researchers will need to screen tens of thousands of cognitively healthy older adults to identify the thousands of individuals eligible to enroll in prevention trials (4). Additionally, the number of trials in affected individuals and their care partners continues to rise. In 2019, there were 156 trials for the treatment of AD, an increase from 2018 (5). Notably, this number does not include observational studies, which are also important to understanding $\mathrm{AD}$ and developing new interventions to treat or prevent it. We use the term "AD-focused studies" to capture these different study types (e.g., clinical trials, observational studies, etc.) and participant populations (e.g., preclinical, mild cognitive impairment (MCI), AD dementia, etc.). The recruitment goals for these $\mathrm{AD}$-focused studies confront the $\mathrm{AD}$ field with a daunting challenge. In the US, regardless of disease area, the vast majority $(85-90 \%)$ of studies experience significant delays in recruitment and enrollment (6). Nearly one-third of clinical trials under-enroll, and only $7 \%$ meet their target enrollment number on time (7). Numerous factors contribute to these difficulties. Recruitment is time-consuming, sometimes taking years to meet target sample sizes. This is in large part because screen failure rates for trials can reach as high as $85 \%$, chiefly due to inclusion criteria. For example, in the 
case of AD-related studies, requiring presence of an $\mathrm{AD}$ biomarker or participants to have a specific genetic risk factor (8). Improving recruitment methods has become a critical priority for the AD field (8-12).

As the number of AD-focused studies increase there is a growing need for (1) increased awareness of research participation opportunities and (2) quick and efficient mechanisms to contact, characterize, and refer potentially eligible participants to studies (13). Initiatives such as the National Plan to Address AD call for greater attention to "increasing enrollment into clinical trials and other clinical research." An outgrowth of the National Plan is the National Strategy for Recruitment and Participation in Alzheimer's Disease Clinical Research which enumerated four goals, one of which focused specifically on the need to "build and improve research infrastructure of registries in order to recruit and retain more and more diverse qualified study participants" (13).

Participant recruitment registries are tools designed to reach out to, identify, characterize, and refer potentially eligible participants to studies, often with the goal of minimizing the percentage of people who screen fail or are otherwise found to be ineligible. In the US and globally, several AD-focused participant recruitment registries are being used at the local, regional, and national level (14-24). Each of these registries approach participant recruitment and engagement differently and the field is still gathering data on best practices to design and conduct recruitment registries in order to help accelerate enrollment into AD prevention-focused studies and trials $(12,13)$.

Here we describe the rationale, design and execution of, as well as enrollment metrics, member demographics, and key lessons learned from the Alzheimer's Prevention Registry (APR). The APR is a large internetbased participant recruitment registry developed by the Banner Alzheimer's Institute (BAI) researchers, leaders of the Alzheimer's Prevention Initiative (API) program. Between 2009-2011, API leaders vetted with academic advisors and stakeholders initial designs for $\mathrm{AD}$ prevention trials in autosomal dominant $\mathrm{AD}$ (ADAD) and APOE4 homozygote populations; the APR was developed as a result of these discussions and a small group of academic advisors formed the APR Executive Committee who provided input and guidance during the early years of its development and launch. The initial design of the APR leveraged our experience leading the state-wide, paper-based Arizona Alzheimer's Registry (AAR) (25) and was inspired by other large, internetbased participant recruitment registries (e.g., Army of Women, ResearchMatch, Fox Trial Finder) (26). The initial objectives were to create an efficient participant recruitment registry that would go beyond the needs of the API trials and help recruit for a range of AD prevention-focused studies that were, at the time, in initial stages of planning and not yet ready to enroll participants. Since the launch of the APR, and due to the still limited number of $\mathrm{AD}$ prevention trials, the program has expanded its reach to help with recruitment for a range of AD-focused studies and occasionally, studies focused on other related dementias or aging and cognition.

\section{Methods}

\section{APR Overview}

Individuals age 18 and older are eligible to join the APR via the website www.endALZnow.org (NCT02022943). The age range was selected to allow younger adults the option to join the APR and share information and study opportunities with family and friends. The APR was determined to not be research by the Institutional Review Board (IRB) based on federal regulation 45 CFR 46 and associated guidance. Although individuals do not provide consent when joining the APR, they do agree to the APR's privacy policy and are informed about BAI's Notice of Privacy Practices (Health Insurance Portability and Accountability Act [HIPAA]).

In 2006, prior to the development of the APR, the Arizona Alzheimer's Consortium (AAC) created the AAR which was then led by BAI. The AAR has been described previously (25). In brief, participation in the AAR was by open invitation to adults in Arizona aged 18 and older. Those interested provided consent and completed a written questionnaire. A subset of Registrants underwent telephone cognitive assessment. Referral to AAC sites for study opportunities was based on Registrants' medical history, telephone cognitive assessment, and research interests. Between 2006 and 2011, 1257 people consented to the AAR, most of whom were cognitively unimpaired at a time when most AD-focused studies were enrolling individuals with cognitive impairment. The AAR proved to be operationally burdensome, requiring data entry from written questionnaires and staff to contact participants by telephone to administer cognitive assessments. These assessments were partially intended as engagement and retention tools for participants, however, due to budget constraints, not all underwent testing, and for those who did, the administration was intermittent. The infrastructure and experiences gained from the AAR served as the prototype for the APR.

\section{APR Website and Member Experience}

The APR website launched in May 2012. Within six months it became apparent that the initial website design, including requiring members to create an account using a username and password, made the process to join the APR too cumbersome. In addition, the initial website was optimized for viewing on traditional desktop computer rather than being "mobile friendly" for viewing on a smartphone or tablet. Lastly, the "call to action" 
messaging on the APR website was confusing, leading website visitors to believe that it was a request for monetary donations. To better understand APR members' use of the website, in 2013 we invited APR members to complete a seven-question survey. The survey consisted of 5 Likert scale questions and 2 open response questions. Results from this survey, along with best practices and lessons learned from other registries and online recruitment/enrollment programs, were used to guide the first website redesign, which went live in July 2013 and was the first step in making the website mobile friendly.

After this first redesign, we used A/B tests to determine which changes to make to the APR website to maximize an outcome of interest, such as increasing enrollment into the APR, by randomly showing website visitors a "control" or "variation" message and then measuring which version was more effective for the intended outcome (27). A/B tests were conducted in October 2013 (testing the APR description/call to action on the website landing page), May 2014 (again testing the APR description/call to action on the website landing page), and September 2014 (testing what member contact and demographic information to collect on the main landing page versus a secondary page).

\section{Recruitment and Enrollment into the APR}

We used several recruitment strategies and tactics to raise awareness about the APR and enroll individuals into the program, including community talks, brochures, paid social media advertisements, and earned media coverage. The frequency in which these have occurred has varied since the APR's launch. Source of enrollment is tracked via Urchin Tracking Module (UTM) codes and stored in the APR database; individuals are not asked where they heard about the APR. To check for accuracy, select UTM codes are checked for accuracy against reports from advertising partner. In 2015, we conducted a paid awareness campaign in partnership with an online advocacy community over a three-month period, asking their community members to electronically sign a petition stating they support AD prevention research (the petition was conceptual and not sent to anyone); those who signed were enrolled automatically in the APR. In 2015, we began a paid social media advertising campaign on platforms including Facebook to help raise awareness about the APR and its GeneMatch program (20); this campaign has run intermittently since 2015. Unlike the online petition campaign, the paid social media campaign directed individuals to the APR website where they could enroll. Funding amounts for these paid campaigns varied from year to year.

\section{APR Member Engagement and Retention}

Multiple strategies were used to help members stay engaged and connected with the APR. Members received email newsletters (titled The Alzheimer's Prevention Bulletin [APB]) highlighting information about AD prevention research. Initially the APB was emailed to members on a quarterly basis but based on the results from the 2013 survey it was moved to monthly in 2014. Also, in 2014 we began sending a caregiver-focused newsletter to APR members who indicated that they are caring for someone with $\mathrm{AD}$ or other dementia. Since 2015, APR members have been able to manage their email subscription preferences directly via the APR website, giving members the ability to select the types of newsletters and study opportunity emails (e.g., prevention studies, studies for people with memory impairment, etc.) they would like to receive and unsubscribe at any point in time. Prior to 2015, APR members managed their email subscriptions via the email footer (e.g., selecting "unsubscribe" at the bottom of the email). In 2017, we added the caregiver newsletter option to the newsletter subscription list, making it available to any APR member who wished to receive it (i.e., they did not need to indicate that they are caring for someone with AD or other dementia). In 2018, we began including a brief, typically one- to three-question, survey in the APB every other month to provide readers an opportunity to express opinions about various AD-related topics. The survey results are shared with readers on the months without a survey.

In 2014, we implemented what is commonly referred to as a "drip email campaign" after a person joined the APR. These emails, sent at prespecified times after enrollment, acknowledged the person's signup, described the APR, and provided information about study opportunities. The drip campaign emails have evolved over the years in terms of their format, content, number, and duration. In 2017 we expanded the drip campaign to include an anniversary email, thanking the person for being an APR member for another year. The anniversary email provided the member with the opportunity to update their APR profile and a reminder to update their email newsletter subscription preferences.

In 2016, we began a re-engagement campaign as a mechanism to reach out to APR members who had not opened one of our emails in the past six-months ("unengaged members"). As part of this campaign, and following email marketing best practices for email list "hygiene" (e.g., to help ensure APR emails are delivered to members' inboxes and are not marked as "spam" or "junk"), we sent up to four emails to "unengaged members" reminding them about the APR and providing instructions if they wanted to stay enrolled in the APR or wish to be removed. If no action was taken after the fourth email, their enrollment was deactivated and they no longer received emails from the APR. As with the 
newsletters and drip campaign emails, the re-engagement campaign emails evolved over time, incorporating email list hygiene best practices from email marketing advisors (see Acknowledgements). In 2017, we changed email platform providers. Only members who opened an APR email within the past 6 months were transferred to the new platform, though their contact information remained in the APR database.

\section{Identifying Studies to Promote to APR Members}

APR staff searched publicly available websites (e.g., clinicaltrials.gov) on a regular basis to identify newly enrolling AD-focused studies and attempted to contact the sponsor or investigator team to discuss notifying APR members about the study. In addition, the APR team staffed an information booth at AD-focused scientific conferences, such as the Alzheimer's Association International Conference (AAIC), to raise awareness about the APR as a recruitment resource to researchers and study sponsors. Study investigators and sponsor teams also contacted the APR team by email or by completing a form on the APR website to inquire about how to list their study on the APR. The APR team collected relevant information such as study design, enrollment criteria, and IRB-approved recruitment materials that were reviewed by the APR Study Review Committee for goodness-of-fit for APR members.

\section{Connecting APR Members to AD-focused Studies}

The APR used two main methods for notifying members about study opportunities, a dedicated "Study Opportunities" section of the website and emails to APR members. In April 2014, we launched the first version of the "Study Opportunities" section of the website. The goal was to create an actionable part of the website so that when visitors come to the website, they can connect with a study opportunity immediately and the APR, in theory, could begin collecting metrics for study referrals. This section contained original, lay-friendly descriptions of the study opportunity (rather than pull information directly from other websites such as clinicaltrials.gov) and the contact information for the study coordinator (or other relevant person/website) was shown after the website visitor (APR member or website visitor who found the APR through a search engine or other means of organic traffic) clicked "Learn More", allowing the visitor to contact the enrolling study directly. The study description was approved by the enrolling study's IRB. Importantly, the APR did not exchange Personally Identifiable Information (PII) or other sensitive information with the enrolling study, since website visitors contacted study staff directly.
In July 2017, we redesigned this section of the website, renaming it "Find a Study." The redesigned section allowed website visitors to search for studies by study type (e.g., online study, observational study, clinical trial, etc.), by enrollment criterion (e.g., their age), keyword search, and/or by location (e.g., zip code or country). Over time, the design was refined, allowing website visitors to search for studies enrolling people with or without memory impairment. Between July 2018 and June 2019, the "Find a Study" section was updated to include a "contact form" for studies rather than listing the study coordinator's contact information. An individual interested in a study was asked to complete the form with their name, email address and phone number, review and acknowledge the study's eligibility criteria and authorize the APR to share their contact information with the enrolling study team. The APR team provided the enrolling study with a dashboard for tracking referrals. Under this new model, studies and/or sponsors were required to execute a data sharing agreement with BAI due to the transfer of PII.

As noted previously, the APR also used email communication with members to connect them with study opportunities. Beginning in 2014, in conjunction with the launch of the "Study Opportunities" section of the website, we began sending specific email campaigns to APR members notifying them when new study opportunities are available. We worked directly with the study/researcher/sponsor to design an email campaign that met their recruitment objectives. The campaigns ranged from small, targeted emails to APR members based on demographic information provided at signup (e.g., age, location) to large, "spread the word" campaigns that requested APR members' assistance to tell their friends and family about a study opportunity. Regardless of the size of the campaign, the emails included a Uniform Resource Locator [URL] hyperlink to the specific study listing on the APR website where they are provided with more information about the study and the study coordinator's contact information. The hyperlink contains a tracking mechanism (via UTM codes), providing limited enrollment metrics to the APR and the enrolling study.

\section{Data Analyses}

Recruitment and enrollment into the APR are ongoing. The current report includes data collected as of December 1, 2019. A/B tests were conducted and analyzed using Optimizely testing software and Optimizely's Stats Engine using a two-tailed sequential likelihood ratio test with false discovery rate controls to calculate statistical significance while minimizing false declarations. 


\section{Results}

\section{APR Member Demographics}

As of December 1, 2019, 346,661 people had joined the APR. Member demographic and recruitment sources are shown in Table 1. Since the website designed evolved over time, and members have not always been required to answer all questions, the sample sizes for each question are provided. Members have a mean age of 63.3 (SD 11.7 ) years, $75 \%$ are female, $94 \%$ self-report being cognitively unimpaired, $50 \%$ have a family history of $\mathrm{AD}$ or other dementia, and of those who provide race and ethnicity, $76 \%$ identify as non-Hispanic white. Of the four recruitment / enrollment tactics, paid social media advertising campaigns resulted in the most people joining the APR (39\%), followed closely by people visiting the APR website directly (e.g., learning about the APR in news article, being referred by a friend, attending a community lecture, etc.) (32\%).

\begin{tabular}{|c|c|}
\hline Age, mean $(\mathrm{SD})(\mathrm{n}=295,384)$ & $63.3(11.7)$ \\
\hline \multicolumn{2}{|l|}{$\operatorname{Sex}(n=303,412)$} \\
\hline Female & $75 \%$ \\
\hline Male & $25 \%$ \\
\hline \multicolumn{2}{|l|}{ Cog status $(n=159,169)$} \\
\hline Cognitively Unimpaired & $94 \%$ \\
\hline Cognitively Impaired & $6 \%$ \\
\hline \multicolumn{2}{|l|}{ Family history of AD or other dementia $(n=140,195)$} \\
\hline Yes & $50 \%$ \\
\hline No & $20 \%$ \\
\hline Unsure & $12 \%$ \\
\hline Prefer not to answer & $18 \%$ \\
\hline \multicolumn{2}{|l|}{ Race and ethnicity ${ }^{*}(\mathrm{n}=207,447)$} \\
\hline White & $76 \%$ \\
\hline Hispanic or Latino & $3 \%$ \\
\hline African American & $2 \%$ \\
\hline Asian & $1 \%$ \\
\hline Other & $1 \%$ \\
\hline More than one race selected & $3 \%$ \\
\hline Prefer not to answer & $13 \%$ \\
\hline \multicolumn{2}{|l|}{ Recruitment / enrollment source $(n=346,660)$} \\
\hline Visited APR website directly or referred from other website & $36 \%$ \\
\hline Paid online advocacy petition & $25 \%$ \\
\hline Paid social media (e.g., Facebook) & $39 \%$ \\
\hline
\end{tabular}

* participants are able select multiple options, only those reported by $0.3 \%$ or more of participants are listed

\section{APR Website and Member Experience}

934 (9\%) of APR members responded to the 2013 survey (Section 2.2). Topline results from the open response questions indicated that APR members wanted more frequent email communication with the latest news (communication had been quarterly email newsletters) and the APR signup processes needed to be simplified with fewer "clicks."

The October 2013 A/B test found that the variation landing page would increase annual enrollment by $8 \%$. The May 2014 A/B test found that the variation landing page would increase annual enrollment by $11 \%$. The September 2014 A/B test did not find a difference between the control and variation, leading us to conclude that we could collect additional contact and demographic information at the first step of enrollment without negatively impacting signups while helping with data cleanliness.

\section{APR Member Engagement and Retention}

In 2019, the average APB email open rate was $45 \%$ (compared to nonprofit healthcare industry average of $16 \%$ ); average email click rate was $24 \%$ (compared to the industry average of $1.6 \%$ ) (28). In 2019, response rates to the brief surveys in the APB ranged from $4.1 \%-10 \%$ among those members who opened the email.

As of December 1, 2019, 86,175 people were considered "actively engaged" members of the APR. In 2017, just prior to when we changed email platforms, the APR had 268,194 members, of whom 85,790 had opened an APR email within the past 6 months. As a result, we only transferred 85,790 to the new email platform. The email addresses from the remaining 182,404 members were not transferred to the new platform but their information remained in the APR database. Since this time, approximately 54,000 members (a mixture of members transferred to the new platform and members who joined after the email platform transition) have been added to the re-engagement campaign on the new email platform, and nearly 10,000 (18.5\%) have been re-engaged and remained enrolled in the APR. Examining re-engagement "failure" rates by source of enrollment, $46 \%$ of those who joined via online advocacy community petition were not able to re-engaged successfully, followed by $37 \%$ of those who joined after seeing a paid social media advertisement, followed by $17 \%$ of those who joined by visiting the APR website directly or were referred by another source. Other members are considered unengaged because they either unsubscribed from receiving APR emails, marked APR emails as spam (and therefore no longer receive APR emails), or provided an invalid email address during enrollment. Since the launch of the APR, approximately $15 \%$ of members unsubscribed from APR emails. Examining unsubscribing by source of enrollment, $17 \%$ of those who joined by visiting the 
APR website directly or were referred by another source unsubscribed, followed by $16 \%$ of those who joined by online community / petition, followed by $12 \%$ who joined after seeing a paid social media advertisement.

\section{Connecting APR Members to AD-focused Studies}

As of December 1, 2019, the APR helped recruit for 82 AD-focused studies. New studies are being added on an ongoing basis to the "Find a Study" page of the website. As mentioned previously, only anecdotal data about APR member enrollment into in-person studies is available. Based on UTM data, APR has helped to enroll 10,005 participants into the Alzheimer's Prevention Trials (APT) Webstudy, 6,559 into the Brain Health Registry [BHR], and 950 into AlzU.org. Seven studies are currently utilizing the "contact form" recruitment model and 250 referrals have been sent to study staff thus far. Future publications will use data from the "contact form" to report on the effectiveness of APR for accelerating recruitment and enrollment into AD-focused studies.

\section{APR as a Foundation for other Enrollment Initiatives}

The APR served as a foundation for GeneMatch, a novel, trial-independent research enrollment program led by the API team at BAI, designed to recruit and refer cognitively healthy adults to AD prevention studies based in part on their APOE test results (NCT02564692) (20). GeneMatch was launched as a program of the APR in 2015 and as of April 2019, had enrolled just over 90,000 participants. In addition, we have shared our experience developing and leading large-scale recruitment registries to help others accomplish shared and complementary goals. This includes participating in the Dementia Research Recruitment Platform Global Collaborative (APR/GeneMatch [USA], BHR [USA], Hersenonderzoek [Netherlands], Join Dementia Research [UK], TrialMatch [USA], and StepUp for Dementia Research [Australia]), sharing data and lessons learned with the Global Alzheimer's Platform (GAP), and participating in the development of the National Institute on Aging (NIA) National Strategy for Recruitment and Participation in Alzheimer's and Related Dementias Clinical Research (13). In addition, we have helped with technological advances, including providing the Frontotemporal Dementia Disorders Registry (FTDDR) with the ability to use all software, code, and learnings of the APR for their program.

\section{Discussion}

The APR, launched in 2012, is a large, internet-based, participant recruitment registry for AD-focused studies, having enrolled over 346,000 members and helped 82 studies try to meet their enrollment goals. The APR was created at a time when several AD prevention trials were on the horizon, but not yet ready to begin recruiting participants. The initial objectives were to create an efficient participant recruitment registry that would go beyond the needs of the API trials and help recruit for a range of $\mathrm{AD}$ prevention-focused studies that were, at the time, in initial stages of planning and not yet ready to enroll participants. As a result, the initial design of the APR focused on providing members with news and information about $\mathrm{AD}$ and prevention research to keep them engaged and retained until studies began recruitment. Since its launch, and due to the still limited number of AD prevention trials, the APR has expanded its reach to help with recruitment for a range of AD-focused studies and occasionally, studies focused on other related dementias or aging and cognition. Along the way, the APR has provided a foundation for other efforts (e.g., GeneMatch), partnered with other national and international efforts to share learning and develop strategies to accelerate enrollment into AD-focused studies, and provided technological assistance to other registries.

The APR website and enrollment process have evolved since 2012. For example, based on learnings from the AAR, the APR was designed intentionally to collect minimal contact and demographic information at enrollment. The manner in which members are presented with request(s) to provide this information changed over time. The initial website design presented all the requested contact and demographic information on one page, requiring people to scroll down the webpage. This layout was conducive to completing enrollment process on desktop or laptop computers, but not mobile devices such as a smartphone or tablet. The first redesign made the process simpler, requiring just an email address to enroll in the APR, and then asked for the remaining information (e.g., name, year of birth, zip/postal code, family history, etc.) on a subsequent page. While this made it quite easy for people to join the APR, we lacked key pieces of information to help connect members to studies (e.g., age, zip/postal code), not all members answered all questions, and the approach affected data cleanliness (i.e., if members shared an email address). Over a series of A/B tests, we were able to land on a middle ground in which we collected a few key pieces of information initially (email address, first and last name, zip/postal code, country, and year of birth), and then presented members with a secondary webpage requesting additional information (e.g., diagnosis of cognitive impairment, family history of dementia, etc.), allowing members to select "prefer not to answer" (as 
opposed to letting them skip answering the question), and the opportunity to manage directly their email newsletter subscriptions. That said, the APR has incomplete demographic profiles for some members, particularly those who enrolled in the early years.

We created the APR with the initial goal of connecting members to AD prevention study opportunities. However, when the APR was launched in 2012, few prevention studies were recruiting participants. As a result, we needed to identify other mechanisms to keep members engaged and connected to the APR so that when such study opportunities became available, there was a large community of individuals ready to be notified. Based on the 2013 survey, we focused our efforts on two main areas: email newsletters and website content. Over the years, we have refined our approach to the email newsletters, transitioning from quarterly to monthly distribution. We continue to strive to make the content appropriate for the general public, not scientists or researchers. The primary APR newsletter, the APB, continues to perform well compared to the healthcare industry standard (28).

As more study opportunities became available, it became apparent that we needed to modify the APR website to make it easier for members to search for study opportunities We developed a "Find a Study" page on the APR website which allows anyone (not just those enrolled in APR) to see all studies for which APR is helping to recruit as well as filter by key criteria such as location, study type, and age eligibility. Rather than pulling the study information from another website, such as clinicaltrials.gov, we developed a Study Opportunity description template which contains high level information about the study design and eligibility criteria. These lay-friendly Study Opportunity descriptions are written jointly by the APR team and recruiting study (or sponsor) and then submitted to the recruiting study's IRB for approval. Until recently, the Study Opportunity description provided the contact information for the study (e.g., contact information for a study coordinator or link to study website) if a person was interested in learning more about the study and/or participating. However, this model did not allow APR to track referrals and or obtain accurate metrics of success for accelerating enrollment into study. As a result, in late 2019 we instituted the "Contact Form" model for new studies listing on APR. This allows interested individuals to give authorization to the APR to transfer their contact information to the enrolling study team via a secure dashboard (the dashboard also allows the enrolling study to track prospective participant referrals). The "Contact Form" model will be offered to studies already listed on the APR (i.e., existing studies) beginning in 2020. Moving forward, the APR will be able to provide more accurate referral metrics.

In addition to sharing information about recruiting studies on the APR website "Find a Study" page, we send announcements about study opportunities to members by email. The APR team works closely with the recruiting study/researcher/sponsor to develop an email campaign to meet their recruitment needs, ranging from a single email to APR members residing in a small radius from the study site and who might be eligible for a study based on their profile, to larger "spread the word" email campaigns to all APR members. The email contains a hyperlink which takes the person to the APR website for a full description of the study and information about next steps if they want to learn more about the study. In addition to sending emails about study opportunities new to APR members, beginning in 2019, the APR began sending members email notifications about studies for which they may be newly eligible (e.g., they now meet the study's age eligibility).

APR has used a variety of recruitment strategies and tactics to enroll members, such as community talks, earned media (i.e., news articles), and paid social media advertisements. Paid online advocacy community petitions and social media advertisements have resulted in the largest numbers of enrollees, although a sizeable percentage unsubscribe from email communications or are unable to be re-engaged successfully. Once APR can accurately track members' interest in studies then we will also be able to examine whether the source of enrollment into the APR is a factor in members' willingness to consider study opportunities as well as the return on investment for the different recruitment strategies and tactics.

Despite using a variety of recruitment strategies and tactics, APR members are predominantly female and self-report being non-Hispanic, white, similar to reports from other internet-based recruitment registries (16). This may be the result of a combination of many factors including the design of and language on the APR website as well as the recruitment strategies and tactics used (13). In addition, women are more likely than men to search for health information online compared, even though men and women are equally likely to have internet access and go online (29). More needs to be done to better understand the barriers and facilitators to enrollment for men and underrepresented racial and ethnic groups as well as understand whether women, in their "health information gathering role" are sharing information from the APR with male family members and friends. In addition, a concerted effort is needed to understand why a sizeable percentage of members prefer not to provide their race/ethnicity during initial enrollment, perhaps adapting strategies found to be effective at a local level to internet-based registries (3032). Identification and removal of these potential barriers, as well as implementation of new recruitment solutions is critically important to meet the goal of enrolling diverse populations into $\mathrm{AD}$ prevention trials (33).

We acknowledge several limitations of the APR. By design, the APR collects minimal information from 
members and does not assess their cognitive functioning, relying instead and on self-reported information provided at enrollment with the option to update at enrollment anniversaries. As a result, some members' profiles may be inaccurate and there may be cases in which a person joins the APR more than once using different email addresses. For these and other reasons, the APR encourages members to review study inclusion criteria and emphasizes to study sites and sponsors the importance of prescreening referrals from the APR. The APR no longer requires members to create an account by establishing an APR username and password. This feature was removed in 2013 because it posed difficulty to members, although with usernames and passwords becoming increasingly common, we are considering reintroducing it as an optional feature in the future. Another limitation is that APR members are not representative of the general population. All participants must have an email address to join the APR. This requirement is a potential barrier for individuals who do not have access to or use email on a routine basis. Moreover, APR members are not representative of the general population with regard to sex, race or ethnicity. Separate efforts are underway to better understand how to communicate the importance of participating in AD-focused studies to men and underrepresented racial and ethnic populations, as well studies to understand the impact the APR website design may have on enrollment of people from diverse backgrounds. The APR is also only available in English due to the staffing requirements needed if the program were to be made available in other languages. For example, in addition to needing to translate all content on the website content and in the newsletters, we would need bilingual staff available to answer members' phone calls and emails. Moreover, there is concern that offering the APR in languages other than English would create false expectations for the availability of study opportunities for non-English speakers in the US. The APR will continue to monitor this and will adapt as needed.

Despite these and other limitations, APR has demonstrated its ability to enroll hundreds of thousands of adults into an internet-based, participant recruitment registry for $\mathrm{AD}$-focused studies, keep members engaged, and help a large number of studies try to meet their enrollment goals. Member engagement and retention continue to be key areas of focus as well as implementing mechanisms that allow the APR to track its effectiveness at helping investigators effectively and efficiently meet their enrollment goals. The efforts of the APR and its GeneMatch program, along with complementary efforts from other local (e.g., Butler Alzheimer's Prevention Registry, North Carolina Brain Health Registry, University of California Irvine [UCI] Consent-toContact [C2C], Wisconsin Registry for Alzheimer's Prevention [WRAP]), national (e.g., APT Webstudy, BHR, GAP, MindCrowd, TrialMatch), and international
(Hersenonderzoek [Netherlands], Join Dementia Research [UK], StepUp for Dementia Research [Australia]) recruitment registry programs have the potential to accelerate enrollment into the growing number of AD-focused studies, thereby helping to advance AD research in ways that would not otherwise be possible.

Acknowledgements: We are grateful for the support of our past and current partners and colleagues at Banner Alzheimer's Institute, The Reis Group, Innolyst and Provoc. We appreciate the support and guidance of the APR Executive Committee: Paul Aisen, Marilyn Albert, Maria Carrillo (ex officio), Meryl Comer, Jeffrey Cummings, Jennifer Manly, Ronald Petersen, Nina Silverberg (ex officio), Reisa Sperling, Gabriel Strobel, and Michael Weiner.

Funding: This work is supported by grants from the National Institute on Aging (R01 AG063954 [JBL], P30 AG19610 [EMR]). The Alzheimer's Prevention Registry has been supported by the Alzheimer's Association, Banner Alzheimer's Foundation, Flinn Foundation, Geoffrey Beene Gives Back Alzheimer's Initiative, GHR Foundation, and the state of Arizona (Arizona Alzheimer's Consortium).

Conflicts of interest: Jessica Langbaum, Nellie High, Cassandra Kettenhoven, Eric Reiman and Pierre Tariot: full employees of Banner Health. Jodie Nichols: no conflicts of interest.

Ethical Standards: The APR was determined to not be research by the IRB Although individuals do not provide consent when joining the APR, they do agree to the APR's privacy policy and are informed about BAI's Notice of Privacy Practices, including HIPAA.

Open Access: This article is distributed under the terms of the Creative Commons Attribution 4.0 International License (http://creativecommons.org/ licenses/by/4.0/), which permits use, duplication, adaptation, distribution and reproduction in any medium or format, as long as you give appropriate credit to the original author(s) and the source, provide a link to the Creative Commons license and indicate if changes were made.

\section{References}

1. Wimo A, Guerchet M, Ali GC, et al. The worldwide costs of dementia 2015 and comparisons with 2010. Alzheimers Dement. 2017;13(1):1-7

2. Alzheimer's Association. 2018 Alzheimer's Disease Facts and Figures Alzheimers Dement 2018;14(3):367-429

3. Brookmeyer R, Gray S, Kawas C. Projections of Alzheimer's disease in the United States and the public health impact of delaying disease onset. Am.J.Public Health 1998;88(9):1337-42

4. Alber J, Lee AKW, Menard W, Monast D, Salloway SP. Recruitment of At-Risk Participants for Clinical Trials: A Major Paradigm Shift for Alzheimer's Disease Prevention. J Prev Alzheimers Dis 2017;4(4):213-4

5. Cummings J, Lee G, Ritter A, Sabbagh M, Zhong K. Alzheimer's disease drug development pipeline: 2019. Alzheimers Dement (N.Y.) 2019;5:272-93

6. Dowling NM, Olson N, Mish T, Kaprakattu P, Gleason C. A model for the design and implementation of a participant recruitment registry for clinical studies of older adults. Clin.Trials 2012;9(2):204-14

7. Strasser JE, Cola PA, Rosenblum D. Evaluating various areas of process improvement in an effort to improve clinical research: discussions from the 2012 Clinical Translational Science Award (CTSA) Clinical Research Management workshop. Clin.Transl.Sci. 2013;6(4):317-20

8. Grill JD, Galvin JE. Facilitating Alzheimer disease research recruitment Alzheimer Dis.Assoc.Disord. 2014;28(1):1-8

9. Schneider LS. Recruitment methods for United States Alzheimer disease prevention trials. J Nutr.Health Aging 2012;16(4):331-5

10. Grill JD, Karlawish J. Addressing the challenges to successful recruitment and retention in Alzheimer's disease clinical trials. Alzheimers.Res.Ther 2010;2(6):34

11. Vellas B, Hampel H, Rouge-Bugat ME, et al. Alzheimer's disease therapeutic trials: EU/US Task Force report on recruitment, retention, and methodology. J Nutr.Health Aging 2012;16(4):339-45

12. Aisen P, Touchon J, Andrieu S, et al. Registries and cohorts to accelerate early phase Alzheimer's trials. A report from the E.U./U.S. Clinical Trials in Alzheimer's Disease Task Force. J Prev Alz Dis 2016;3(2):68-74

13. Together we make the difference: National strategy for recruitment and participation in Alzheimer's and related dementias clinical research National Institutes of Health NIoA. 2018 Oct.

14. Krysinska K, Sachdev PS, Breitner J, et al. Dementia registries around the globe and their applications: A systematic review. Alzheimers Dement. 2017;13(9):1031-47

15. Grill JD, Hoang D, Gillen DL, et al. Constructing a Local Potential Participant 
Registry to Improve Alzheimer's Disease Clinical Research Recruitment. J Alzheimers Dis. 2018;63(3):1055-63

16. Weiner MW, Nosheny R, Camacho M, et al. The Brain Health Registry: An internet-based platform for recruitment, assessment, and longitudinal monitoring of participants for neuroscience studies. Alzheimers Dement. 2018;14(8):1063-76

17. Chadiha LA, Washington OG, Lichtenberg PA, et al. Building a registry of research volunteers among older urban African Americans: recruitment processes and outcomes from a community-based partnership. Gerontologist. 2011;51 Suppl 1:S106-S115

18. Johnson SC, Koscik RL, Jonaitis EM, et al. The Wisconsin Registry for Alzheimer's Prevention: A review of findings and current directions. Alzheimers Dement.(Amst.) 2018;10:130-42

19. Vermunt L, Veal CD, Ter ML, et al. European Prevention of Alzheimer's Dementia Registry: Recruitment and prescreening approach for a longitudinal cohort and prevention trials. Alzheimers Dement. 2018;14(6):837-42

20. Langbaum JB, Karlawish J, Roberts JS, et al. GeneMatch: a novel recruitment registry using at-home APOE genotyping to enhance referrals to Alzheimer's prevention studies. Alzheimer's and Dementia 2019;15(4):515-24

21. Rios-Romenets S, Lopez H, Lopez L, et al. The Colombian Alzheimer's Prevention Registry. Alzheimer's \& Dementia 2017;13(5):602-5

22. Larsen ME, Curry L, Mastellos N, et al. Development of the CHARIOT Research Register for the Prevention of Alzheimer's Dementia and Other Late Onset Neurodegenerative Diseases. PLoS.One. 2015;10(11):e0141806

23. Lim YY, Yassi N, Bransby L, Properzi M, Buckley R. The Healthy Brain Project: An Online Platform for the Recruitment, Assessment, and Monitoring of Middle-Aged Adults at Risk of Developing Alzheimer's Disease. J Alzheimers Dis 2019;68(3):1211-28
24. Juaristi GE, Dening KH. Promoting participation of people with dementia in research. Nurs.Stand. 2016;30(39):38-43

25. Saunders KT, Langbaum JB, Holt CJ, et al. Arizona Alzheimer's Registry: strategy and outcomes of a statewide research recruitment registry. J Prev Alz Dis 2014;1(2):74-9

26. IOM (Institute of Medicine). Models for Public Engagement. In Public Engagement and Clinical Trials: New Models and Disruptive Technologies: Workshop Summary. Washington, DC: The National Academies Press; 2012.

27. Online Controlled Experiments and A/B Tests Kohavi R, Longbotham R. Springer; 2016.

28. Benchmarks M+R. 2016. mrbenchmarks.com

29. Profiles of Health Information Seekers Pew Research Center. Washington, DC: Pew Research Center's Internet \& American Life Project; 2011 Feb 1.

30. Williams MM, Scharff DP, Mathews KJ, et al. Barriers and facilitators of African American participation in Alzheimer disease biomarker research. Alzheimer Dis.Assoc.Disord. 2010;24 Suppl:S24-S29

31. Hinton L, Carter K, Reed BR, et al. Recruitment of a community-based cohort for research on diversity and risk of dementia. Alzheimer Dis.Assoc.Disord. 2010;24(3):234-41

32. Dilworth-Anderson P, Williams SW. Recruitment and retention strategies for longitudinal African American caregiving research: the Family Caregiving Project. J Aging.Health. 2004;16(5 Suppl):137S-56S

33. Watson JL, Ryan L, Silverberg N, Cahan V, Bernard MA. Obstacles and opportunities in Alzheimer's clinical trial recruitment. Health Aff.(Millwood.) 2014;33(4):574-9 